\title{
Postural Hypo-Reactivity in Autism is Contingent on Development and Visual Environment: A Fully Immersive Virtual Reality Study
}

\author{
Selma Greffou • Armando Bertone • \\ Eva-Maria Hahler • Jean-Marie Hanssens • \\ Laurent Mottron · Jocelyn Faubert
}

Published online: 13 July 2011

(C) The Author(s) 2011. This article is published with open access at Springerlink.com

\begin{abstract}
Although atypical motor behaviors have been associated with autism, investigations regarding their possible origins are scarce. This study assessed the visual and vestibular components involved in atypical postural reactivity in autism. Postural reactivity and stability were measured for younger (12-15 years) and older (16-33 years) autistic participants in response to a virtual tunnel oscillating at different frequencies. At the highest oscillation frequency, younger autistic participants showed significantly less instability compared to younger typicallydeveloping participants; no such group differences were evidenced for older participants. Additionally, no significant differences in postural behavior were found between all 4 groups when presented with static or without visual information. Results confirm that postural hypo-reactivity to visual information is present in autism, but is contingent on both visual environment and development.
\end{abstract}

S. Greffou · E.-M. Hahler · J.-M. Hanssens $\cdot$ J. Faubert $(\bowtie)$ Visual Psychophysics and Perception Laboratory, School of Optometry, University of Montreal, 3744 Jean-Brillant street, Montreal, QC H3T 1P1, Canada

e-mail: jocelyn.faubert@umontreal.ca

\section{A. Bertone}

Perceptual Neuroscience Laboratory for Autism and

Development, Montreal, Canada

\section{A. Bertone - L. Mottron}

Department of Psychiatry, University of Montreal, Montreal, Canada

A. Bertone $\cdot$ L. Mottron

University of Montreal Center of Excellence for Pervasive

Developmental Disorders, Montreal, Canada
Keywords Autism - Posture - Development . Sensorimotor $\cdot$ Virtual reality $\cdot$ Motion perception

\section{Introduction}

Autism is a behaviorally variant phenotype with a neurogenetic basis characterized by atypical communication and social interaction, co-occurring with restricted interests and repetitive behaviours (American Psychological Association 1994). Visual information processing is also atypical in autism, defined by a "perceptual signature" characterized by superior performances on perceptual and cognitive tasks where local or detailed processing of spatial information is advantageous, and by a decreased ability or optional processing for complex types of information requiring either integrative, dynamic or global analysis (see Mottron and Burack 2001; Mottron et al. 2006); Dakin and Frith 2005; Behrmann et al. 2006; Bertone and Faubert 2006; Happe and Frith 2006; Simmons et al. 2009; Bertone et al. 2010; for reviews).

Posture is regulated via the integration of signals originating from three afferent sensory systems: the somatosensory, the vestibular and the visual systems (Peterka and Benolken 1995; Nolan et al. 2005). These signals are then used by the cortex and cerebellum to produce an appropriate motor output within a changing visual environment. A deficit in any of these systems can affect posture and balance. Given autism's "perceptual signature", altered postural regulation is expected since visual information processing is involved in several visually-contingent behaviors, including maintaining posture, or balance. Although abnormalities of motor behavior, most often described as "associated symptoms", (i.e., either clumsiness, fine/gross motor deficits, apraxia, alterations in motor 
milestone development, etc....) have been well documented in autism (Teitelbaum et al. 1998; Ghaziuddin and Butler 1998; Ming et al. 2007), relatively few studies have directly assessed either balance and/or postural reactivity in autism. In one such study, Gepner et al. (1995) reported an attenuation of reactivity to a radiating full-field optic flow stimulus, which typically induces the illusory perception of self motion, particularly for fast visual motion (see Gepner and Mestre 2002a). This study involved a small group of five young children with autism whose ages ranged between 4 and 7 years (and whose intellectual level of functioning was not documented). Gepner and colleagues concluded that persons with autism, especially those with low functioning autism (LFA), were insensitive to dynamic visual information with regards to posture compared to control participants, which probably originated from an impairment in motion perception; a lack of attention to stimuli was also suggested. However, it can also be argued that postural attenuation might have resulted from a motor functioning impairment in the autism group (particularly in the LFA group), resulting in inadequate motor output despite appropriate sensory functioning. These and other results related to the perception of both social and nonsocial information (Gepner and Mestre 2002a) have been used to propose that a "rapid visual motion integration deficit" (Gepner and Mestre 2002b), and more recently, a "temporo-spatial processing disorder" (Gepner and Féron 2009) may underlie postural anomalies in autism.

Subsequent studies assessing posture in autism have manipulated proprioceptive input by having participants stand on foam (or not) under different visual conditions. For example, Molloy et al. (2003) demonstrated that on average, autistic children were less stable when standing passively and blindfolded, thus eliminating visual cues, whether or not proprioceptive information was modified. Reflecting over-reliance on visual input for maintaining balance in the autism/ASD group, this result was interpreted as evidence for a multi-modal dysfunction in the integration of information originating from visual, somatosensory, and vestibular afferences in autism. Using a larger sample of 79 high-functioning autistic participants aged between 5 and 52 years, Minshew et al. (2004) demonstrated that the postural stability of autistic participants was reduced when proprioceptive input was disrupted by a sway-referenced platform. In addition, results demonstrated that postural control started to develop later in the autism group (12 years of age compared to 5 years in the control group) and never reached neuro-typical, adult-like levels. These results were also interpreted as evidence for both delayed and underdeveloped postural control in autism, and also argued to result from a deficit of multimodal sensory integration between the different neural systems underlying postural control in autism.
Taken together, all of these results suggest atypical or underdeveloped postural control in autism that may derive from a multi-modal sensory integration deficit, either resulting from impaired complex motion perception (Gepner et al. 1995), or from atypical integrative functioning between any of the subsystems involved in postural control (Molloy et al. 2003; Minshew et al. 2004). In order to isolate the subsystems underlying postural control in autism, we have assessed postural behavior in response to immersive visual environments differing only as a function of oscillation frequency, while the other sub-systems, namely the somatosensory and vestibular systems, were kept constant. A fully immersive virtual reality approach was used to measure postural reactivity and stability in autism relative to a sway-inducing virtual tunnel (see "Methods" section) oscillating at three different frequencies (see Greffou et al. 2008; Piponnier et al. 2009). Postural behavior was assessed above and below the age of 16 years (participants included in either 12-15 years, or 16-33 years age groups) in order to assess whether postural behavior differs as a function of development. The age ranges used to create our groups were chosen based on previous findings demonstrating that postural reactivity to the exact same visual environment used in the present study reached adult-like levels at 16 years of age for neurotypical participants (Greffou et al. 2008). In addition, the immersive nature of our virtual reality approach minimizes possible confounding variables such as inattentiveness to the visual environment (Gepner et al. 1995) for both autistic and control participants. This approach also allows for the manipulation of visual environment characteristics (tunnel oscillation frequency) on a continuum, rather than on a categorical level (present or absent).

\section{Methods}

\section{Participants}

The autistic and typically-developing (TD) participant groups were placed in either of two age groups: $12-15$ yearolds and 16-33 year-olds. Therefore, the study included a total of four groups: a $12-15$ year-old autism group $(n=8$; $\mathrm{M}=13.0 \pm 1.3$ year-old), a $12-15$ year-old TD group ( $n=11 ; \quad \mathrm{M}=13.6 \pm 1.6$ year-old $), \quad$ a $16-33$ year-old autism group $(n=8 ; \mathrm{M}=21.0 \pm 5.9$ year-old $)$, and a 16-33 year-old TD group $(n=23 ; \mathrm{M}=23.0 \pm 5.4$ yearold).

\section{Autism Group}

Sixteen individuals (3F; $13 \mathrm{M})$ with autism were randomly extracted from Rivière-des-Prairies' hospital database and 
invited to participate in this study. Autism was diagnosed using the Autism Diagnosis Interview-Revised (Lord et al. 1994) and the Autism Diagnosis Observation Schedule (Lord et al. 2000), both of which were conducted by a trained clinician-researcher (LM) who obtained reliability on these instruments. Thirteen of the participants with autism scored above the ADI and ADOS cut-off in the three relevant areas for diagnosis (social, communication, restricted interest and repetitive behaviours). One autistic participant did not score above cut off in the Communication domain on both instruments; and two participants were administered an expert (but not standardized) clinical DSM-IV diagnosis of autism following a direct observation based on the ADOS procedure. Participants with other developmental DSM-IV Axis 1 diagnoses, except hyperactivity and language disorders, potentially relevant Axis 3 diagnoses, non-corrected-to-normal vision (20/20 Snellen acuity for both eyes) and without adequate stereoscopic vision were excluded from enrolment in this study. Two autistic participants (one in each age group) were taking Concerta (a slow-release psychostimulant used to manage ADHD) at the time of testing as part of their daily routine. All participants in the autism group had full-scale Wechsler IQ scores in the normal range (12-15 year-olds: $98.75 \pm$ $16.2 ; 16-33$ year-olds; $101.13 \pm 12.0$ ).

\section{Typically Developing Group}

Performance of participants with autism was compared to that of thirty-four typically developing (TD) individuals. TD individuals were recruited by word of mouth in the community, and none of them reported problems when screened by a semi-structured interview documenting history of psychiatric or neurological condition, learning disabilities, family history $\left(1^{\circ}\right)$ of mood disorders, pervasive developmental disorders or schizophrenia, defective vision or audition and intake of medication. All participants were informed of the goals of the study and nature of the tasks and their consent was obtained. All participants were compensated monetarily for their time. Testing commenced after the ethics committees at the Rivière-desPrairies Hospital and at the University of Montreal (where the testing took place) approved of the study.

\section{Apparatus}

Postural reactivity to visual information was assessed using a fully immersive virtual environment (CAVE system, Fakespace $\left.^{\mathrm{TM}}\right)$. The CAVE is an $8 \times 8 \times 8$ feet room including three canvas walls (one frontal and two laterals) and an epoxy floor, all serving as surfaces for the projection of images (Fig. 1). The resolution of each surface image

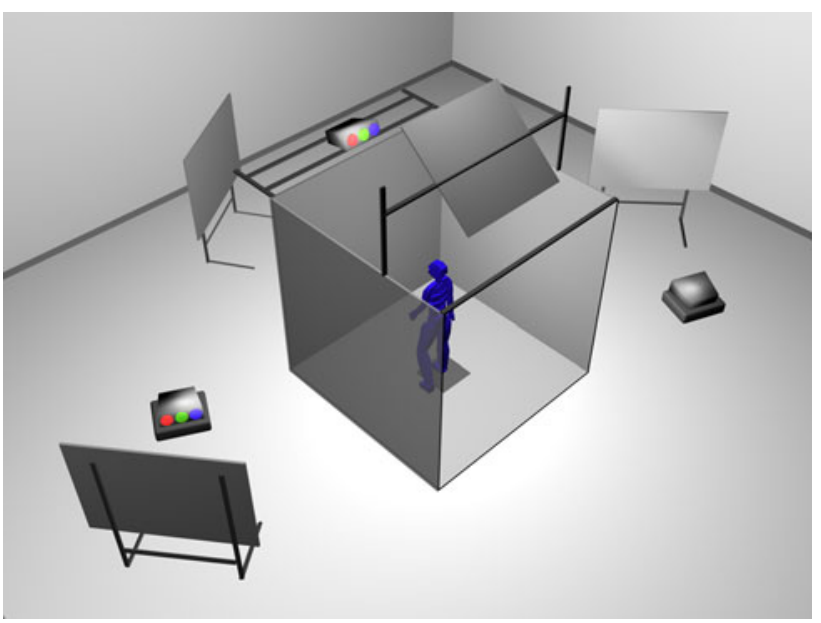

Fig. 1 The CAVE is an $8 \times 8 \times 8$ feet room that includes three walls (one frontal and two lateral) and a floor that all serve as surfaces for the projection of images

was $1,280 \times 1,024$ pixels, and was generated by Marquee Ultra 8500 projectors.

The CAVE was under the control of a SGI ONYX $3200^{\circledR}$ computer equipped with two Infinite Reality II graphics cards and a magnetic motion tracker system (Flock-of-Birds ${ }^{\circledR}$ ) measuring postural reactivity by registering body movement at the head level. A previous study conducted in the laboratory (Faubert and Allard 2004) along with some pilot data using the same setup as was used in the present study showed that the measures taken at the head level (sensor positioned on the stereo goggles) yielded comparable results to those obtained when sensors were positioned on the lower back (lumbar 2-3). This demonstrates that, at least under our present conditions, the postural response of our participants resembled that of an inversed pendulum motion pattern. We have therefore decided to use only the sensor at the head level so as to avoid adding methodologically superfluous and potentially invasive lumbar sensors. The polarized stereoscopic goggles (Crystal Eyes ${ }^{\circledR}$, StereoGraphics Corporation; Fig. 2)

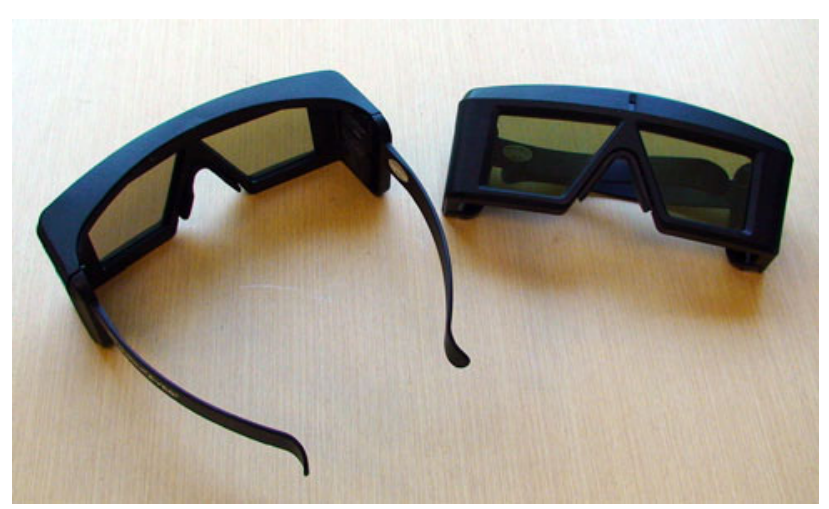

Fig. 2 The stereoscopic goggles 
were equipped with a magnetic motion sensor allowing for the precise tracking and measurement of their motion (thus, the motion of participants). Three dimensional vision being the result of the computation of two different images (one from each eye) by the brain, wearing of stereoscopic goggles allowed for the alternating occlusion of the left and right eyes at a high frequency $(96 \mathrm{~Hz})$. This occlusion was synchronized with the projection frequency on the screens, resulting in a three-dimensional perception of the environment. For more details on the equipment used, please visit the following website: http://vision.opto.umontreal.ca/ English/Techno/CAVE.html.

\section{Procedure}

Participants were first familiarized with the virtual environment. Visual acuity and stereoscopic vision were then assessed without glasses (using a Snellen eye-chart and a Random Dots Stereo-acuity Test). Participants were then asked to wear the stereoscopic goggles, and were positioned at $1.50 \mathrm{~m}$ from the CAVE's central wall with shoes off, feet together, and arms crossed. This position was chosen in order to minimize the use of individual strategies from the limbs to maintain posture, and helped maximize the effect of the visual stimulation (Kawakita et al. 2000). For all conditions, participants were asked to fixate a red dot located at the horizon. Behavioral information was recorded as participants simply stood in the virtual reality environment while they were presented with the visual stimulation.

\section{Experimental Paradigm}

The postural reactivity of participants was assessed using the "Virtual Tunnel Paradigm" (Fig. 3; for a video of this paradigm: http://vision.opto.umontreal.ca/Techno/CAVE. html). The tunnel had an inner texture made of a checkerboard pattern, where each high-contrast square was scaled for linear perspective (for a detailed description, see Greffou et al. 2008; Piponnier et al. 2009). Two types of visual environments, dynamic and static, were used in this study.

For the dynamic condition, the simulated motion of the tunnel was defined by an anterior-posterior (front-back) sinusoidal translation motion oscillating around the participants at 3 different frequencies: $0.125,0.25$, or $0.5 \mathrm{~Hz}$ (for further details on the choice of these frequencies or on the physical properties of the tunnel, please refer to Greffou et al. 2008). For each frequency, participants performed three $68 \mathrm{~s}$ trials, resulting in a total of 9 dynamic trials, presented in a pseudo-random order. The initial frequency was randomly selected and each consecutive presentation

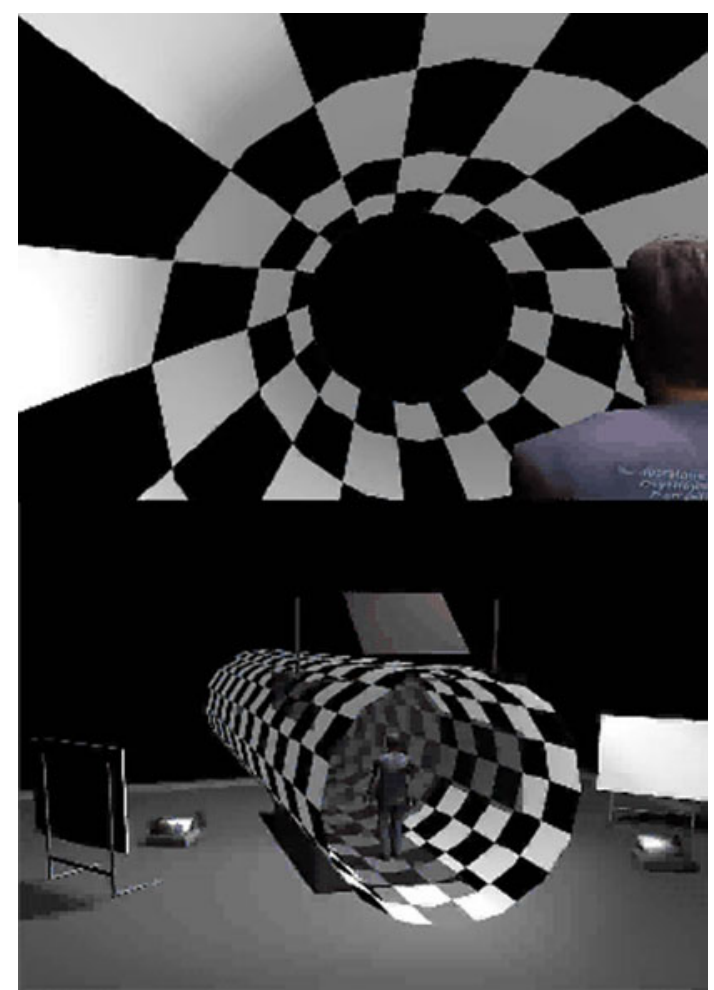

Fig. 3 The virtual tunnel paradigm (http://vision.opto.umontreal.ca)

of a given frequency was always separated by at least one presentation of each of the two other frequencies. Static conditions served as control conditions, thus allowing us to separate the effect of dynamic visual stimulation on postural reactivity from that of static visual stimulation and spontaneous sway. In the static tunnel condition, participants had to fixate a red dot presented at the horizon during two $68 \mathrm{~s}$ trials, while standing in the virtual tunnel in its static state $(0 \mathrm{~Hz}$, i.e. motionless $)$. Since the structure, dimension and texture of the tunnel were identical in both dynamic and static conditions, the unique variable differentiating the two conditions was its apparent motion. For the eyes closed condition, participants were asked to position their heads as if they were fixating the horizon, but had their eyes closed. This condition was added to measure the extent to which visual input, whether dynamic or static, affected postural reactivity. In summary, all participants performed thirteen $68 \mathrm{~s}$ trials in the following order; 2 static tunnel trials, 9 dynamic tunnel trials, and 2 eyesclosed trials. A trial was considered incomplete if either a participant lost balance during the trial (i.e., he or she could not remain standing with feet together), or asked for the trial to be stopped. If a participant was unable to complete two out of the three dynamic trials for a given oscillation frequency, his/her data was excluded from statistical analyses. 


\section{Behavioral Measures}

Changes in posture were monitored using two measures: Body Sway (BS) and Postural Perturbations (PP), (see Greffou et al. 2008). BS is the anterior-posterior displacement of a participant as a function of the virtual tunnel's oscillation frequency. Due to the variation of height as a function of age group, angular displacement (Fig. 4) was used as the dependent measure of postural reactivity as opposed to linear displacement. PP is defined as the root mean squared (RMS) of total body velocity in the horizontal plane (i.e., anterior-posterior " $z$ axis" and medial-lateral displacements " $x$ axis") in angles per second (Faubert and Allard 2004; Greffou et al. 2008; Piponnier et al. 2009). This measure was used in order to quantify postural perturbations induced by the visual stimuli. The PP measure is distinct from the BS one in that it is not computed relative to a single specific frequency; rather, it reflects body instability at all frequencies minus the one at which the tunnel "moves" during the trial of interest.

\section{Results}

\section{Statistical Analyses}

Separate analyses were performed for Body Sway (BS) and Postural Perturbations (PP) given that each of these two variables represents a different element of postural reactivity: BS reflecting synchronicity to stimulation, and PP reflecting general instability. In the dynamic tunnel condition, results from 3 trials were averaged for each

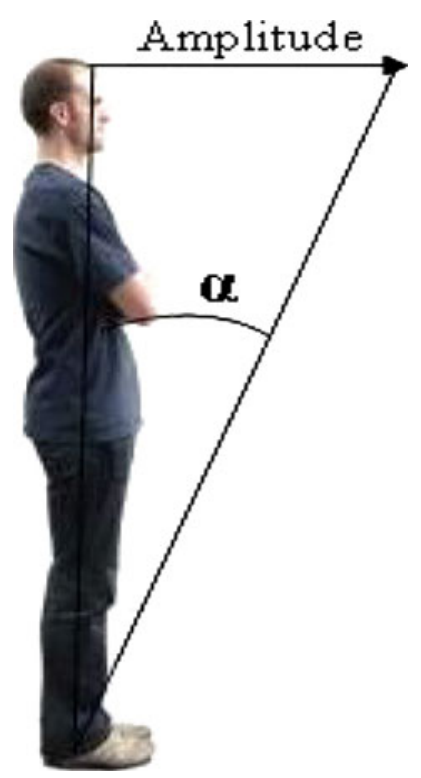

Fig. 4 Angular displacement of a person frequency, resulting in one value per frequency for each participant. The same principle was applied for the control conditions: 2 trials per frequency were averaged for each participant; therefore, each participant had only one score per frequency, per condition (dynamic tunnel, eyes closed and static tunnel). Raw scores were converted to log values. Note that the data of one autistic participant in the 12-15 year-old group was removed from statistical analyses as he was unable to complete all of the $0.25 \mathrm{~Hz}$ trials due to dizziness and to technical problems during testing.

\section{Body Sway Analyses (BS)}

An Age (2) $\times$ Group (2) $\times$ Frequency (3) mixed factorial analysis of variance (ANOVA) was performed for the dynamic condition. A significant main effect of Age $(F(1,45)=13.01, p=.001, \eta 2=.224)$ and a non-significant Group (autism vs. TD) $\times$ Frequency interaction $(F[1.84,82.90]=0.52, \quad p=.58) \quad$ demonstrated that younger participants (12-15 year-old) swayed more than older participants across all frequencies regardless of whether they belonged to the autism or TD groups. Moreover, a 2 (Group) × 2 (Age) $\times 3$ (Frequency) mixed factorial ANOVA revealed a significant three-way interaction $F[1.84,82.90]=3.67, p=.033, \eta 2=.075)$; pairwise comparisons using Bonferroni correction revealed that amongst the autistic group, the 12-15 year-olds manifested significantly more Body Sway than did the adults for the 0.125 and $0.25 \mathrm{~Hz}$. The same was true of the TD groups, where 12-15 year-olds showed significantly more body sway than did the adults but this time for the 0.25 and $0.50 \mathrm{~Hz}$. A between-group difference in Body Sway for younger participants failed to reach statistical significance for all of the frequencies, but a trend was noted at $0.50 \mathrm{~Hz}$ where younger participants with autism swayed less than younger TD ones (See Fig. 5).

Age (2) $\times$ Group (2) $\times$ Frequency (3) mixed factorial analyses of variance were also performed for both of the Eyes Closed (EC) and Static Tunnel (ST) conditions

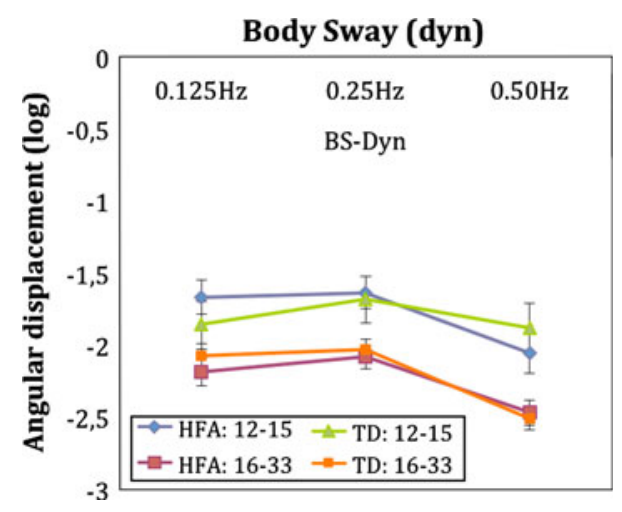

Fig. 5 Body sway as a function of frequency and group 
(Figures not shown). For EC, no significant main effect of Group $(F[1,45]=1.27, p=.27)$ nor a significant threeway interaction $(F[2,90]=1.19, p=.31)$ were found. Likewise, for the Static Tunnel condition (ST), no significant main effect of Group $(F[1,45]=0.072, p=.79)$ or a significant Age $\times$ Group $\times$ Frequency interaction were found $(F[2,90]=1.31, p=.28)$. Finally, an ANOVA comparing Eyes Closed and Static Tunnel, where BS scores were collapsed across frequencies, showed that participants were less reactive during the Static Tunnel condition as compared to during the Eyes Closed condition $(F[1,6]=7.02, p=.038)$ regardless of frequency, as no significant Condition $\times$ Frequency interaction was found $(F[2,12]=3.31, p=0.072)$.

Postural Perturbations Analyses (PP)

Age (2) $\times$ Group (2) $\times$ Frequency (3) mixed factorial analyses of variance were performed. For the dynamic condition, a significant main effect of Age $(F[1,45]=$ $20.16, p=.000, \eta 2=.309)$ demonstrated that younger participants (12-15 year-old) were less stable than older participants across all frequencies regardless of whether they belonged to the autism or TD groups. Furthermore, a 2 (group) $\times 2$ (age) $\times 3$ (Frequency) mixed factorial ANOVA revealed a significant three-way interaction $(F[1.67,75.13]=4.23, p=.024, \eta 2=.086)$; pairwise comparisons using Bonferroni correction revealed that at $0.50 \mathrm{~Hz}$, the $12-15$ year-old autism group $(\mathrm{M}=-1.62$, $\mathrm{SD}=.18)$ manifested significantly more postural stability than did the 12-15 year-old TD group $(\mathrm{M}=-1.42$, $\mathrm{SD}=.32),(t(16)=2.08, p=.043)$; as was previously mentioned, the same tendency was observed for BS although it failed to reach statistical significance for $0.50 \mathrm{~Hz}$ (See Fig. 6).

Age (2) $\times$ Group (2) $\times$ Frequency (3) mixed factorial analyses of variance were also performed for both the EC

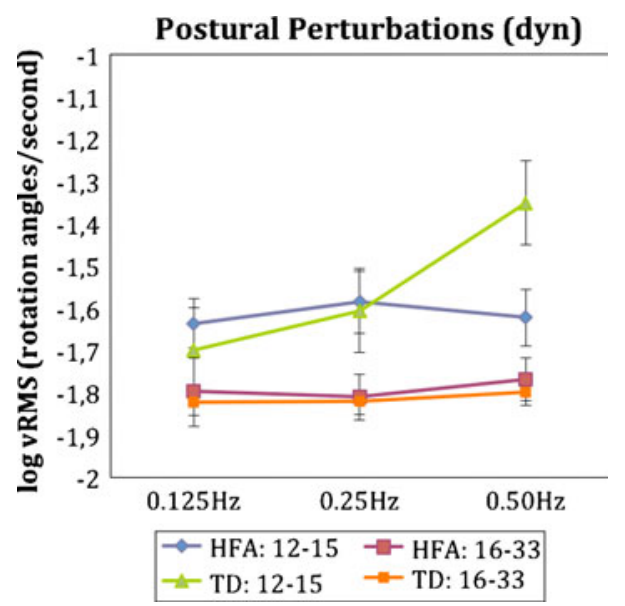

Fig. 6 Postural perturbations as a function of frequency and group and ST conditions (Figures not shown). For EC, no significant main effects of Group was revealed $(F[1,45]=$ $.029, p=.86)$ nor was a significant three-way interaction $(F[1.07,48.07]=.36, p=.57)$. Similarly, for ST no significant main effect of $\operatorname{Group}(F[1,45]=1.11, p=.30)$ or a significant Age $\times$ Group $\times$ Frequency interaction were evidenced $(F[1.84,82.56]=.44, p=.63)$. Finally, an ANOVA comparing Eyes Closed and Static Tunnel, where PP scores were collapsed across frequencies, showed that participants, as was the case for BS, were more stable during the Static Tunnel condition as compared to during the Eyes Closed condition $(F[1,6]=5.10, p=.065)$ regardless of frequency, as no significant Condition $\times$ Frequency interaction was found $(F[1.2,7.2]=2.80$, $p=0.14)$.

\section{Discussion}

Although atypical motor behaviors are often described as associated behavioral symptoms of autism, their etiology remains unknown. Given the altered visually-related information processing in autism, an important component of motor regulation, we assessed the visual and vestibular components involved in postural reactivity in autism by measuring perturbation and body sway induced by a virtual tunnel oscillating at different frequencies for younger and older participants with autism. Compared to typicallydeveloping participants, younger participants with autism were hypo-reactive showing significantly less postural perturbation to the sway-inducing virtual tunnel only at the highest oscillation frequency $(0.50 \mathrm{~Hz})$. No significant differences in postural reactivity were found between the two older groups across the three frequencies tested in the dynamic condition. In addition, postural behavior did not differ between groups when immersed in control environments, where afferent visual input was either present and static (immersed in static tunnel) or absent (eyes closed condition). These results suggest that hypo-reactivity to visual-inducing information in autism is contingent on both visual environment (ex: speed of visual stimuli) and development (chronological age), and probably not the result of a vestibular dysfunction; if such were the case, between groups differences would be found throughout all of the experimental conditions, particularly Eyes Closed, where the vestibular system is more strongly solicited.

Atypical Postural Behavior And Dynamic Information Processing in Autism

As was mentioned in the Introduction, the paradigm used in the present study is novel in that postural behavior in autism was not simply assessed as a function of whether 
afferent visual input was present or not (i.e., eyes-closed vs. eyes-opened). We assessed the implication of vestibular and visual components of postural behavior by measuring how this behavior was differentially affected by manipulating the dynamicity (tunnel frequency oscillation) of the visual environment wherein the participants were immersed. Results demonstrated that for younger participants with autism (12-16 years), hypo-reactivity (i.e., less postural perturbation) to a sway-inducing visual environment was only manifested for the highest oscillation frequency $(0.50 \mathrm{~Hz})$; between-group differences were not demonstrated for slower oscillation frequencies. Moreover, a similar trend was noted for the Body Sway measure where young participants with autism swayed less than the young TD; body sway being mostly a measure of synchronicity to stimuli (see "Methods" section), this implies that our younger autistic participants seem not to have synchronized normally to the fastest stimulation frequency whereas they were able to do so for lower frequencies. In summary, autistic participants were able to integrate and translate most sensory information into an appropriate motor response under most experimental conditions except when the processing and integration of fast visual stimuli was required.

These results are consistent with the "visual-motion integration deficit" (Gepner and Mestre 2002a) and/or the "temporo-spatial processing disorder" (Gepner and Féron 2009) hypotheses proposing that atypical postural reactivity in autism is specific to fast moving visual stimulation. In general, these hypotheses are based on findings of decreased postural reactivity of autistic participants to a 2-dimensional flow-field, defined by an oscillating circularly-symmetric, frequency-modulated concentric grating (Gepner et al. 1995), particularly for fast visual motion (Gepner and Mestre 2002a). In these two studies (the latter described as a replication and extension of the former), the oscillation frequency - or driving frequency - of the grating was set at $0.2 \mathrm{~Hz}$, resulting in different local angular velocities across the stimuli since the spatial frequency of the concentric rings defining the flow field decreased from center of focus of expansion/contraction. It is important to note that the effect of velocity on reactivity was computed by transforming (Fast Fourier Transformation or FFT) center-of-pressure measures into the fore-aft sway axis into components at each local angular velocity (ranging from $6^{\circ}$ to $100 \%$ s). Therefore, the interpretations of Gepner and colleagues are based on postural reactivity findings with respect to local (peak) angular velocities, and not to the consequence of manipulating the overall velocity of the sway-inducing flow-fields. It is also worth noting that although the hypotheses advanced by Gepner and Mestre were based on results originating from rather small sample sizes (i.e., Gepner and Mestre 2002a: autistic disorder, $n=3$; Asperger $n=3$; neurotypical, $n=9$ ), they are consistent with ours as only young participants with an autistic disorder diagnosis (and not Asperger) demonstrated differential reactivity to visual information.

In the present study, three different dynamic driving frequencies were assessed $(0.125,0.25$ and $0.5 \mathrm{~Hz})$. By assessing postural behavior under different levels of dynamic visual stimulation, and not only comparing postural behavior in dynamic versus static environments, perceptual versus visuo-motor origins of atypical postural reactivity in our autism group were dissociated. The finding that postural behavior in the autism group was comparable to that of controls for the lower velocities argues against the suggestion that atypical postural behavior in autism is due to motion perception impairments (Gepner et al. 1995). Specifically, a motion perception deficit would predict atypical reactivity across all oscillation frequencies assessed, since the visual environment induced frequencydependant sway for most conditions in the autism group. This finding is especially pertinent since the frequencycontingent autistic behavior occurred within an identical dynamic visual environment in all frequency conditions (except for its velocity level), and cannot be explained by inattention to stimuli, given the immersive character of the virtual visual environment and the small intra-subject variance between the 3 trials at each frequency. In addition, although there is some evidence of motion perception impairments in autism under specific experimental conditions (Bertone et al. 2003; see Bertone and Faubert 2006; Kaiser and Shiffrar 2009 for reviews), it is unlikely that such subtle perceptual deficits alone would translate into the atypical postural behavior observed in this study, given the intensity of the high-contrast information defining the virtual tunnel.

Plausible neural mechanisms contingent on dynamic information processing include the visuo-cerebellar circuits due to their role in the speed and temporal coding of dynamic visual input. Interestingly, visuo-postural miscoupling is representative of a sensory-motor coupling disorder, first described 40 years ago (Ornitz and Ritvo 1968; Ornitz 1974) as a possible etiology of some autistic behaviors (see Ornitz et al. 1985: visuo-vestibular disconnect). In addition, anomalies of cerebello-premotor-motor cortex loops, due to the contribution of both the cerebellum and the basal ganglia to real-time fine-tuning of motor output and to motor learning via their projections to the motor, premotor, prefrontal, temporal and parietal cortices may also be candidate mechanisms that are underdeveloped in autism. This disordered under- or over- visuopostural coupling in children with ASD may partly explain sensory-motor and motor disturbances, such as poor motor coordination, poor or enhanced postural control, and gross or fine motor clumsiness (Ornitz 1974; Damasio and 
Maurer 1978; Kohen-Raz et al. 1992; Leary and Hill 1996; Green et al. 2009 for reviews).

Developmental Trend of Postural Behavior in Autistic and Neurotypical Individuals

Previous results assessing visually-driven postural reactivity during typical development demonstrated that both children and young adolescents show less stability in reaction to dynamic visual scenes (dynamic virtual tunnel, as was used in this study) than adults; they reach adult-like levels between 16 and 19 years of age, suggesting an important transitory period for sensorimotor development (Greffou et al. 2008). In the present study, autistic and nonautistic participants who were 12-15 years of age exhibited more body sway and postural perturbations (vRMS) than did older participants. This finding is in accordance with the developmental trajectory observed in the aforementioned study. In addition, only in this younger age range were between-group differences contingent on the visual environment (oscillation frequency) manifested, suggesting that atypical postural reactivity behavior in autism is most evident before the critical period of sensorimontor development in neurotypical individuals. In another study, Minshew et al. (2004) demonstrated that the postural control of persons with autism aged 5-52 year-old did not begin to improve until the age of 12 years, but never reached adult-like levels. Methodological differences (stimulation and measures) between the Minshew et al. (2004) and the present study may account for discrepancies regarding the transitory periods of sensorimotor development in autism. However, both studies suggest that development is an important component of atypical postural behavior in autism. These findings may be related to the reduced prevalence of motor deficits (fine motor control and programming) in older children with autism spectrum disorder, whether through natural progression, results of interventional therapy, or the combination of the two (Ming et al. 2007). In conclusion, the finding that postural hyporeactivity in autism occurred in the younger autism group when the inducing motion was fastest is suggestive of a delayed development of sensory-motor coupling in autism.

\section{Vestibular and Somatosensory Effects on Postural}

Behavior in Autism

Although direct assessments of vestibular functioning in autism has been relatively limited, studies assessing vestibulo-ocular responses have demonstrated that vestibulorelated autistic dysfunction is most probably due to integrative deficits between the vestibular and other afferent systems (i.e., visual and/or somatosensory), rather than specific deficits to the peripheral vestibular system (Ornitz et al. 1985). This notion is consistent with our findings since a between-group difference in postural behavior (either reactivity or stability) was not evidenced for static conditions. In addition, behavior did not differ across the different static conditions (i.e., static tunnel vs. eyesclosed), suggesting that stability was typical in participants with autism whether or not afferent visual information was available. These results suggest that atypical postural reactivity for our autistic participants did not originate uniquely from a vestibular dysfunction. In addition, the lack of between-group differences for the static conditions (and most dynamic conditions) also suggests that if muscular or morphological differences between autistic and non-autistic participants were present (Leary and Hill 1996; Hallett et al. 1993; Vilensky et al. 1981), they were not significant enough to affect postural behavior under the experimental conditions and paradigm used.

Somatosensory afferent input was kept constant across conditions in this study given that the main goal was to isolate and assess the effect of visual environment on postural behavior (participants stood passively with their shoes off and feet together on a cushionless platform). In a previous study, modifying somatosensory input using a cushioned platform failed to significantly affect postural stability, defined by a sway area covered during a $30 \mathrm{~s}$ trial, in a group of 8 boys with ASD (Molloy et al. 2003). Results from this study also demonstrated that the stability of the ASD group significantly decreased during 'eyesclosed' conditions, regardless of whether somatosensory input was modified or not. This result was interpreted as suggestive of an over-reliance on visual input to maintain balance in the group assessed and is, in general, consistent with a reduced integration between different afferent sensory systems (Molloy et al. 2003; Minshew et al. 2004).

\section{Findings in Relation to the Autistic Behavioral Phenotype}

Although the presence of repetitive behaviors is considered to be a core characteristic of autistic spectrum disorder, there is presently little understanding regarding basic issues such as pathogenesis, purpose, preservation, and ultimately, the management of such behaviors in autism. Nevertheless, hypotheses implicating emotional (BaronCohen et al. 2000), executive (Ozonoff et al. 1991; Joseph and Tager-Flusberg 2004; Hill 2004; see Turner 1999 for reviews) and sensory/perceptual (Rimland 1994; O'Gorman 1967; Delacato 1974; Mottron et al. 2007) origins have been advanced. The latter hypothesis suggests that characteristic repetitive behaviors serve as coping mechanisms by persons with autism in response to an atypically interpreted environment. The present study demonstrated that the postural behavior (passive) of 
autistic participants differed under specific conditions of visual stimulation (i.e., higher oscillation frequencies), suggesting an association between perceptual environment and subsequent behavior. This association can be translated into real-life situations where temporally-changing visual environments are actively produced by behaviors often manifested by persons with autism that include: (a) visual rotation induced by repetitive spinning movements, and (b) the periodic visual stimulation induced by periodic hand or finger movements in the visual field. Whereas spinning behaviours are a reliable part of the autistic phenotype (Bracha et al. 1995), atypical lateral fixations are associated with produced or searched periodic movements (Mottron et al. 2007).

The production of periodic body movements by autistics has generally been interpreted as the semi voluntary behaviour implicating a vestibular input within a framework of atypical sensory modulation (Ornitz 1974). However, our findings suggest that any explanatory model for atypical body movements in autism should consider a possible decoupling between vestibular and visual systems under certain conditions of dynamic visual stimulation. Anecdotally, this suggestion is supported by the frequently reported behavioural observation that prolonged rocking, spinning and whirling behaviours in autism do not result in dizziness (Ornitz 1974; Grandin 1996).

\section{Limitations and Future Directions}

Findings from this study are specific to participants diagnosed with autism who have an IQ comparable to that of typically-developing persons. It is unknown whether this pattern of results transfers across the autism spectrum (Asperger syndrome or Pervasive Developmental Disorder not Otherwise Specified). However, the passive nature of the fully immersive task makes it possible to assess children with limited language and cognitive ability. Future studies could compare different types of dynamic stimuli (e.g. swaying floors), and use non-periodic or unpredictable visual scene movement in order to verify whether abnormalities are manifested in other contexts where efficient visuo-motor integration and complex visual perception is required.

Acknowledgments We would like to thank Autism Speaks Pilot grant to Jocelyn Faubert, Laurent Mottron, and Armando Bertone, and the CFI Leader's fund and NSERC for their grants to Jocelyn Faubert. We would also like to thank greatly all participants and their families for their time and support. These results have been presented by Selma Greffou under the form of a poster at the 7th Annual Meeting for Autism Research in the year of 2008, London, UK; they have also been presented by Armando Bertone under the form of an oral presentation at the 30th European Conference on Visual Perception, Arezzo, Italy.
Open Access This article is distributed under the terms of the Creative Commons Attribution Noncommercial License which permits any noncommercial use, distribution, and reproduction in any medium, provided the original author(s) and source are credited.

\section{References}

American Psychological Association. (1994). Diagnostic and statistical manual of mental disorders (4th ed.). Washington, DC: APA.

Baron-Cohen, S., Ring, H. A., Bullmore, E. T., Wheelwright, S., Ashwin, C., \& Williams, S. C. (2000). The amygdala theory of autism. Neuroscience and Biobehavioral Reviews, 24(3), $355-364$.

Behrmann, M., Thomas, C., \& Humphreys, K. (2006). Seeing it differently: visual processing in autism. Trends in Cognitive Science, 10, 258-264.

Bertone, A., \& Faubert, J. (2006). Demonstrations of decreased sensitivity to complex motion information not enough to propose an autism-specific neural etiology. Journal of Autism and Developmental Disorders, 36, 55-64.

Bertone, A., Hanck, J., Kogan, C. S., Chaudhuri, A., \& Cornish, K. M. (2010). Using perceptual signatures to define and dissociate neural etiology: Autism and fragile $\times$ syndrome as model conditions. Journal of Autism and Developmental Disorders, 40, 1531-1540.

Bertone, A., Mottron, L., Jelenic, P., \& Faubert, J. (2003). Motion perception in autism: A "complex" issue. Journal of Cognitive Neuroscience, 15, 218-225.

Bracha, H. S., Livingston, R., Dykman, K., Edwards, D. R., \& Adam, B. (1995). An automated electronic method for quantifying spinning (circling) in children with autistic disorder. Journal of Neuropsychiatry and Clinical Neuroscience, 7, 213-217.

Dakin, S., \& Frith, U. (2005). Vagaries of visual perception in autism. Neuron, 48, 497-507.

Damasio, A. R., \& Maurer, R. G. (1978). A neurological model of autism. Archives of Neurology, 35, 777-786.

Delacato, C. H. (1974). The ultimate stranger: The autistic child. Garden City, NY: Doubleday.

Faubert, J., \& Allard, R. (2004). Effect of visual distortion on postural balance in a full immersion stereoscopic environment. In A. J. Woods, J. O. Merrit, S. A. Benton, \& M. T. Bolas (Eds.), Stereoscopic displays and virtual reality systems XI (Vol. 5291, pp. 491-500).

Gepner, B., \& Féron, F. (2009). Autism: A world changing too fast for a mis-wired brain? Review. Neuroscience and Behavioral Reviews, 33, 1227-1242.

Gepner, B., \& Mestre, D. R. (2002a). Brief report: postural reactivity to fast visual motion differentiates autistic from children with Asperger syndrome. Journal of Autism and Developmental Disorders, 32, 231-238.

Gepner, B., \& Mestre, D. R. (2002b). Rapid visual-motion integration deficit in autism. Trends in Cognitive Sciences, 6, 455.

Gepner, B., Mestre, D. R., Masson, G., \& Schonen, S. (1995). Postural effects of motion vision in young autstic children. Neuroreport, 6, 1211-1214.

Ghaziuddin, M., \& Butler, E. (1998). Clumsiness in autism and Asperger syndrome: A further report. Journal of Intellectual Disability Research, 42, 43-48.

Grandin, T. (1996). Thinking in pictures and other reports from my life with autism. New York, NY: Vintage Books.

Green, D., Charman, T., Pickles, A., Chandler, S., Loucas, T., Simonoff, E., et al. (2009). Impairment in movement skills of 
children with autistic spectrum disorders. Developmental Medicine and Child Neurology, 51(4), 311-316.

Greffou, S., Bertone, A., Hanssens, J., \& Faubert, J. (2008). Development of visually driven postural reactivity: A fully immersive virtual reality study. Journal of Vision, 8(15), 1-10.

Hallett, M., Lebiedowska, M. K., Thomas, S. L., Stanhope, S. J., Denckla, M. B., \& Rumsey, J. (1993). Locomotion of autistic adults. Archives of Neurology, 50, 1304-1308.

Happe, F., \& Frith, U. (2006). The weak coherence account: detailfocused cognitive style in autism pectrum disorders. Journal of Autism and Developmental Disorders, 36, 5-25.

Hill, E. L. (2004). Executive dysfunction in autism. Trends in Cognitive Sciences, 8(1), 26-32.

Joseph, R. M., \& Tager-Flusberg, H. (2004). The relationship of theory of mind and executive functions to symptom type and severity in children with autism. Development and Psychopathology, 16(1), 137-155.

Kaiser, M. D., \& Shiffrar, M. (2009). The visual perception of motion by observers with autism spectrum disorders: A review and synthesis. Psychonomic Bulletin \& Review, 16, 761-777.

Kawakita, T., Kuno, S., Miyake, Y., \& Watanabe, S. (2000). Body sway induced by depth linear vection in reference to central and peripheral visual field. Japanese Journal of Physiology, 50, 315-321.

Kohen-Raz, R., Volkmar, F. R., \& Cohen, D. J. (1992). Postural control in children with autism. Journal of Autism and Developmental Disorders, 22, 419-432.

Leary, M. R., \& Hill, D. A. (1996). Moving on: Autism and movement disturbance. Mental Retardation, 34, 39-53.

Lord, C., Risi, S., Lambrecht, L., Cook, E. H., Leventhal, B. L., DiLavore, P. C., et al. (2000). The Autism Diagnostic Observation Schedule-Generic: A standard measure of social and communication deficits associated with the spectrum of autism. Journal of Autism and Developmental Disorders, 30, 205-223.

Lord, C., Rutter, M., \& Le Couteur, A. (1994). Autism Diagnostic Interview-Revised: A revised version of a diagnostic interview for caregivers of individuals with possible pervasive developmental disorders. Journal of Autism and Developmental Disorders, 24, 659-685.

Ming, X., Brimacombe, M., \& Wagner, G. C. (2007). Prevalence of motor impairment in autism spectrum disorders. Brain Development, 29, 565-570.

Minshew, N. J., Sung, K., Jones, B. L., \& Furman, J. M. (2004). Underdevelopment of the postural control system in autism. Neurology, 63, 2056-2061.

Molloy, C. A., Dietrich, K. N., \& Bhattacharya, A. (2003). Postural stability in children with autism spectrum disorder. Journal of Autism and Developmental Disorders, 33, 643-652.

Mottron, L., \& Burack, J. (2001). Enhanced perceptual functioning in the development of autism. In J. A. Burack, A. Charman, N. Yirmiya, \& P. R. Zelazo (Eds.), The development of autism:
Perspectives from theory and research (pp. 131-148). Mahwah, NJ: Erlbaum.

Mottron, L., Dawson, M., Soulières, I., Hubert, B., \& Burack, J. (2006). Enhanced perceptual functioning in autism: An updated model, and eight principle of autistic perception. Journal of Autism and Developmental Disorders, 36(1), 27-43.

Mottron, L., Mineau, S., Martel, G., Bernier, C. S., Berthiaume, C., Dawson, M., et al. (2007). Lateral glances toward moving stimuli among young children with autism: Early regulation of locally oriented perception? Developmental Psychopathology, 19, 23-36.

Nolan, L., Grigorenko, A., \& Thorstensson, A. (2005). Balance control: Sex and age differences in 9- to 16-year-olds. Developmental Medicine and Child Neurology, 47, 449-454.

O'Gorman, G. (1967). The nature of childhood autism. London: Butterworths.

Ornitz, E. M. (1974). The modulation of sensory input and motor output in autistic children. Journal of Autism and Childhood Schizophrenia, 4, 197-215.

Ornitz, E. M., Atwell, C. W., Kaplan, A. R., \& Westlake, J. R. (1985). Brain-stem dysfunction in autism: Results of vestibular stimulation. Archives of General Psychiatry, 42, 1018-1025.

Ornitz, E. M., \& Ritvo, E. R. (1968). Neurophysiologic mechanisms underlying perceptual inconstancy in autistic and schizophrenic children. Archives of General Psychiatry, 19, 22-27.

Ozonoff, S., Pennington, B. F., \& Rogers, S. J. (1991). Executive function deficits in high-functioning autistic individuals: relationship to theory of mind. Journal of Child Psychology and Psychiatry, 32(7), 1081-1105.

Peterka, R. J., \& Benolken, M. S. (1995). Role of somatosensory and vestibular cues in attenuating visually induced human postural sway. Experimental Brain Research, 105, 101-110.

Piponnier, J. C., Hanssens, J. M., \& Faubert, J. (2009). Effect of visual field locus and oscillation frequencies on posture control in an ecological environment. Journal of Vision, 9, 1-10.

Rimland, B. (1994). Infantile autism: The syndrome and its implications for a neural theory of behavior. New York, NY: AppletonCentury-Crofts.

Simmons, D. R., Robertson, A. E., McKay, L., Toal, E., McAleer, P., \& Pollick, F. E. (2009). Vision in autism spectrum disorders. Vision Research, 49, 2705-2739.

Teitelbaum, P., Teitelbaum, O., Nye, J., Fryman, J., \& Maurer, R. G. (1998). Movement analysis in infancy may be useful for early diagnosis of autism. Proceedings of the National Academy of Sciences, 95(23), 13982-13987.

Turner, M. A. (1999). Annotation: Repetitive behavior in autism: A review of psychological research. Journal of Child Psychology and Psychiatry, 40, 839-849.

Vilensky, J. A., Damasio, A. R., \& Maurer, R. G. (1981). Gait disturbances in patients with autistic behavior: A preliminary study. Archives of Neurology, 38, 646-649. 